\title{
Prevalence of multidrug-resistant bacteria colonisation among asylum seekers in western Switzerland
}

Journal of Infection Prevention

$1-4$

(C) The Author(s) 2021

Article reuse guidelines:

sagepub.com/journals-permissions DOI: | 0.1 | 777/|757|77420982033

jip.sagepub.com

\author{
Alain Kenfak-Foguena ${ }^{1 * \mathbb{D}}$, Immaculée Nahimana Tessemo ${ }^{2,3 *}$, \\ Claire Bertelli ${ }^{4}$, Laurent Merz ${ }^{5}$, Alain Cometta ${ }^{5}$, Dominique Blanc ${ }^{3}$, \\ Laurence Senn ${ }^{3}$ and Christiane Petignat ${ }^{2,3}$
}

\begin{abstract}
The recent increase of migration to Europe represents a risk of increased the prevalence of multidrug-resistant (MDR) bacteria. We conducted a cross-sectional study among asylum seekers admitted at two hospitals in Switzerland.

Of the 59 patients included, 9 (I 4\%) were colonised by a MDR bacteria, including 5 (8.5\%) methicilin-resistant Staphylococcus aureus (MRSA) and 4 (6.8\%) extended-spectrum beta-lactamase (ESBL)-producing Enterobacteriaceae. No patient carried both ESBL-producing bacteria and MRSA. None of the patients carried a vancomycin-resistant Enterococcus (VRE) or a carbapenem-resistant Enterobacteriaceae (CRE). Colonisation with MDR bacteria was not associated with hospitalisation abroad or recent arrival in Switzerland. Whole genome sequencing analysis allowed us to exclude transmission between patients.
\end{abstract}

The prevalence of MDR bacteria carriage is moderate among asylum seekers in western Switzerland. Further surveillance studies are necessary to determine if there is a risk of dissemination of pathogens into the local population.

\section{Keywords}

Multidrug-resistant bacteria, asylum seekers, Switzerland, Europe, methicilin-resistant Staphylococcus aureus, extendedspectrum beta-lactamases producing Enterobacteriaceae, vancomycin-resistant Enterococcus, carbapenem-resistant Enterobacteriaceae

Date received: 3I August 2020; accepted: 8 November 2020

\section{Introduction}

Multidrug-resistant (MDR) bacteria represent a major threat to public health, with higher prevalence in some parts of the world, especially in low-resource countries. Human migration and food trade contribute to transferresistant bacterial strains from one place to another. The recent increase of migration to Europe thus represents a risk of increased prevalence of MDR bacteria. MDR bacterial infections also represent an issue for migrants' health as they can delay adequate treatment. While increasing data are being published after the recent migration crisis, these usually represent local experiences. Few data are available from the different regions of Switzerland, which otherwise has a low prevalence of MDR bacteria (Nellums et al., 2018): in the range of 5\%-8\% for
'Service of Internal Medicine, Jura Bernois Hospital, Moutier, Switzerland

${ }^{2}$ Cantonal Unit for Infection Control and Prevention, Public Health Service, Lausanne, Switzerland

${ }^{3}$ Service of Hospital preventive medicine, Lausanne University Hospital and University of Lausanne, Lausanne, Switzerland

${ }^{4}$ Institute of Microbiology, Lausanne University Hospital and Lausanne University, Lausanne, Switzerland

${ }^{5}$ Division of internal medicine, Etablissement Hospitalier du Nord Vaudois, Yverdon-les-Bains, Switzerland

*Equal contributors.

Corresponding author:

Alain Kenfak-Foguena, Service of Internal Medicine, Jura Bernois

Hospital, Rue Beausite 49, Moutier, 2740, Switzerland.

Email: alain.kenfak@hjbe.ch 
extended-spectrum beta-lactamase (ESBL)-producing Enterobacteriaceae (Martinez et al., 2019; Pasricha et al., 2013b) and 2\%-5\% for methicilin-resistant Staphylococcus aureus (MRSA) (Harbarth et al., 2005, 2008; Pasricha et al., 2013a). The aim of this study was to assess the prevalence of MDR bacteria carriage among asylum seekers in western Switzerland.

\section{Methods}

We conducted a cross-sectional study among asylum seekers admitted at two hospitals in Switzerland: the Lausanne University Hospital and the Nord Vaudois Regional Hospital. We included all patients administratively tagged as 'asylum seekers (registered in a center for asylum seekers with a specific health insurance card), aged $\geqslant 18$ years and admitted at the hospital within $72 \mathrm{~h}$. Consent was required from patients before inclusion and sample collection. Patients were screened for MRSA, ESBL-producing Enterobacteriaceae, vancomycin-resistant Enterococcus (VRE) and carbapenem-resistant Enterobacteriaceae (CRE). Collected samples (pooled nose, groin, throat for MRSA and rectal swab for VRE, ESBL and CRE) were incubated on selective media and bacteria identification performed using standard procedures including Matrix Assisted Laser Desorption Ionization - Time of Flight (MALDI-TOF) as described previously (Croxatto et al., 2012). Genomic DNA was extracted with the Wizard Genomic DNA Purification kit (Promega, USA). Libraries were prepared using Nextera XT and sequenced on a MiSeq (Illumina, USA). Reads from enterobacterial strains were processed with default parameters of the MetaGenLab diagnostic pipeline (https://github.com/metagenlab/diag pipelines) to perform species assignment and single nucleotide polymorphism (SNP) analyses. Reads from MRSA were analysed with Bionumerics v. 7.6.3 (Applied Maths, Belgium). The study was approved by Vaud state ethical committee (no. 2017-01601).

\section{Results}

We included 59 patients within an 18-month period, from January 2018 to June 2019 (85\% in 2018 and $15 \%$ in 2019), instead of 200 patients originally expected.

The study population had a mean age of 43 years and $46 \%$ were women (Table 1). In total, 26 patients (44\%) had been hospitalised during their migration or in the last year. The duration of stay in Switzerland was in the range of two days to one year. Almost half of the patients $(29 / 59,49 \%)$ originated from Georgia, and other patients came from Afghanistan, Algeria, Angola, Armenia, Congo, Eritrea, Guinea, Iraq, Iran, Lebanon, Libya, Morocco, Somalia, Syria and Venezuela.
Overall, 9 (14\%) patients were colonised by a MDR bacteria, including 5 (8.5\%) MRSA and 4 (6.8\%) ESBL. No patient carried both ESBL-producing bacteria and MRSA. None of the patient carried a VRE or a CRE. Colonisation with MDR bacteria was not associated with hospitalisation abroad or recent $(<3$ months) arrival in Switzerland $(P=0.41)$.

All five MRSA carriers were women living in two different asylum seeker centers and had not been previously hospitalised abroad. Whole genome sequencing (WGS) analysis of the five MRSA strains revealed they belonged to different MLST Sequence Types (ST) (Figure 1). Three of them belonged to $S$. aureus majors Clonal Complex (CC 5, 8 and 30), which include MRSA.

Among the four patients (three men and one woman) positive for ESBL-producing bacteria, 3 (75\%) had been previously hospitalised abroad. Identification of bacterial species using MALDI-ToF indicated that all four patients were colonised by ESBL-producing Klebsiella spp., and one patient had a dual carriage with an ESBL-producing Morganella morganii. WGS analysis allowed for the refining of the taxonomical classification of the Klebsiella spp. as two Klebsiella pneumoniae and two Raoultella ornithinolytica (Figure 1). More than 1000 loci or SNP differences were observed between isolates within the same species, confirming that no direct transmission occurred between these patients.

\section{Discussion}

This study showed the absence of CRE and VRE colonisation, and a $14 \%$ prevalence of MRSA and ESBLs carriage among asylum seekers in western Switzerland, higher than in the local population. Overall, the prevalence of MDR bacteria is moderate, comparable with the Swiss local population for ESBL-producing bacteria (5\%-8\%) and slightly higher for MRSA $(2 \%-5 \%)$ (Fulchini et al., 2019). While another Swiss study reported a mean of $15.7 \%$ of MRSA and $23.6 \%$ of ESBL prevalence during an outbreak in asylum centers in 2015 (Piso et al., 2017), the lower prevalence obtained in the present study may be more representative of baseline asylum seeker colonisation in the absence of outbreaks. Data from other European countries show variable results, according to the country and period of time.

Interestingly, none of the MRSA carriers had been previously hospitalised, while carriage of ESBL tended to be linked to hospitalisation abroad, although not reaching statistical significance owing to the small sample size. In our study, the large number of SNPs on the complete genome allowed us to exclude transmission between patients of MRSA, Klebsiella and Raoultella. Carriage of MRSA may be associated with migrant camp and centre conditions (inadequate sanitation, overcrowding, restricted access to 
Table I. Characteristics of patients.

\begin{tabular}{|c|c|c|c|}
\hline & Total $(\mathrm{n}=59)$ & No carriage $(n=50)$ & MDR carriage $(n=9)$ \\
\hline \multicolumn{4}{|l|}{ Gender } \\
\hline Male & $32(54)$ & $29(58)$ & $3(54.2)$ \\
\hline Female & $27(46)$ & $21(42)$ & $6(45.8)$ \\
\hline Age (years) & $43.3 \pm 15.1$ & $44.1 \pm 14.7$ & $39.0 \pm 17.5$ \\
\hline Duration of stay in Switzerland (days) & $122.0 \pm 127.2(2-421)$ & $122.5 \pm 126.7(2-4 \mid 5)$ & $119.3 \pm 138.9(7-421)$ \\
\hline \multicolumn{4}{|l|}{ Recent hospitalisation abroad } \\
\hline Yes & $27(46)$ & $26(52)$ & $6(66.7)$ \\
\hline No & $32(54)$ & $24(48)$ & $3(33.3)$ \\
\hline \multicolumn{4}{|l|}{ Country of origin } \\
\hline Afghanistan & $3(5.1)$ & $3(6)$ & 0 \\
\hline Algeria & $4(6.8)$ & $4(8)$ & 0 \\
\hline Angola & $\mathrm{I}(\mathrm{I} .7)$ & 0 & $\mathrm{I}(\mathrm{II})$ \\
\hline Armenia & I (I.7) & I (2) & 0 \\
\hline Congo & $2(3.4)$ & I (2) & $\mathrm{I}(\mathrm{II})$ \\
\hline Eritrea & $2(3.4)$ & I (2) & $\mathrm{I}(\mathrm{II})$ \\
\hline Georgia & $29(49.1)$ & $28(56)$ & I (II) \\
\hline Guinea & $3(5.1)$ & $3(6)$ & 0 \\
\hline Iraq & I (I.7) & 0 & I (II) \\
\hline Iran & I (I.7) & I (2) & 0 \\
\hline Lebanon & I (I.I) & 0 & I (II) \\
\hline Libya & I (I.7) & I (2) & 0 \\
\hline Morocco & I (I.7) & I (2) & 0 \\
\hline Somalia & $3(5.1)$ & I (2) & $2(22)$ \\
\hline Syria & $5(8.5)$ & $4(8)$ & I (II) \\
\hline Venezuela & I (I.7) & I (2) & 0 \\
\hline
\end{tabular}

health services), as previously suggested (Nellums et al., 2018). We found no patient colonised with VRE or CRE. Current local recommendations to screen patients who have been hospitalised abroad during the past year, for MDR bacteria carriage, seem adequate in identifying patients carrying ESBLs.

The study was stopped before the planned number of patients could be included due to a decreased inclusion rate, mainly explained by the reduction of new arrival of asylum seekers over the study period. Indeed, in Vaud state, 3142 new seekers have been registered in 2015, 1137 in 2018 and 784 in 2019 (Vaud State Statistics, 2020). A longer inclusion period could itself have included epidemiological changes (countries of origin, migration routes, etc.), potentially leading to different results. Although the study was conducted over 18 months, the small number of participants may bias the representation of MDR prevalence. A nationwide study including more patients over a limited period of time is needed and could help assess MDR prevalence according to the origin and migration routes.

\section{Conclusion}

The prevalence of MDR bacteria carriage is moderate among asylum seekers in western Switzerland. Further surveillance studies are necessary to determine if there is a risk of dissemination of pathogens into the local population. Meanwhile, we do not recommend specific isolation procedure for all asylum seekers in western Switzerland but a targeted screening based on risk factors. 
Figure I. Minimum spanning trees of MDR bacteria. Each circle represents one isolate. The number of SNPs between isolates is reported above the lines for (a) MRSA, (b) K. pneumoniae and (c) R. ornithinolytica. SNPs were identified by mapping sequence reads on one of the assembled genomes as reference for K. pneumoniae and $R$. ornithinolytica. The sequence type is indicated above the circles, when available. MDR, multidrug-resistant; MRSA, methicillin-resistant Staphylococcus aureus; SNP, single nucleotide polymorphism.

(a)

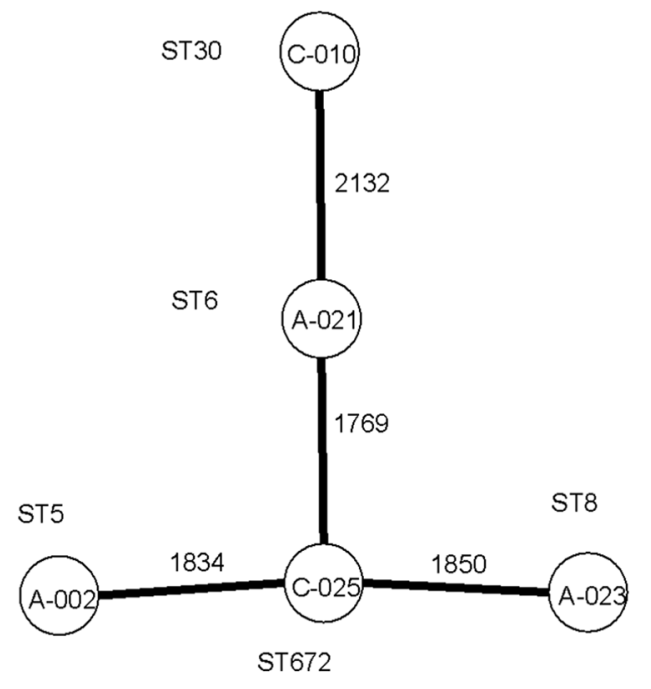

(b)

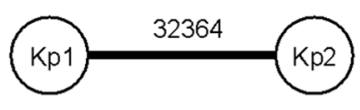

(c)

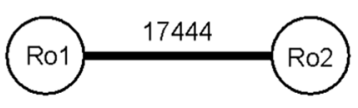

\section{Declaration of conflicting interests}

The author(s) declared no potential conflicts of interest with respect to the research, authorship, and/or publication of this article.

\section{Funding}

The author(s) disclosed receipt of the following financial support for the research, authorship, and/or publication of this article: Public health service of the canton of Vaud

\section{Peer review statement}

Not commissioned; blind peer-reviewed.

\section{ORCID iD}

\section{Alain Kenfak-Foguena (iD https://orcid.org/0000-0002-8213- 2490}

\section{References}

Croxatto A, Prod'hom G and Greub G. (2012) Applications of MALDITOF mass spectrometry in clinical diagnostic microbiology. FEMS Microbiology Reviews 36(2): 380-407.

Fulchini R, Albrich WC, Kronenberg A, Egli A, Kahlert CR, Schlegel $\mathrm{M}$ and Kohler P. (2019) Antibiotic-resistant pathogens in different patient settings and identification of surveillance gaps in Switzerland - a systematic review. Epidemiology \& Infection 147: e259.

Harbarth S, Fankhauser C, Schrenzel J, Christenson J, Gervaz P, BandieraClerc C, Renzi G, Vernaz N, Sax H and Pittet D. (2008) Universal Screening for Methicillin-Resistant Staphylococcus aureus at Hospital
Admission and Nosocomial Infection in Surgical Patients. JAMA 299(10): 1149-1157.

Harbarth S, François P, Schrenzel J, Frankhauser-Rodriguez C, Hugonnet S, Koessler T, Huyghe A and Pittet D. (2005) Community-associated Methicillin-resistant Staphylococcus aureus, Switzerland. Emerging Infectious Diseases 11(6): 962-965.

Martinez AE, Widmer AF, Frei R, Pargger H, Tuchscherer D, Marsch S, Egli A and Tschudin-Sutter S. (2019) ESBL-colonization on ICU admission: impact on subsequent infection, carbapenem consumption and outcome. Infection Control and Hospital Epidemiology 40(4): 408-413.

Nellums LB, Thompson H, Holmes A, Castro-Sanchez E, Otter JA, Norredam M, Friedland JS and Hargreaves S. (2018) Antimicrobial resistance among migrants in Europe: a systematic review and metaanalysis. Lancet Infectious Diseases 18(7): 796-811.

Pasricha J, Harbarth S, Koessler T, Camus V, Schrenzel J, Cohen G, Pittet D, Perrier A and Iten A. (2013a) Methicillin-resistant Staphylococcus aureus risk profiling: who are we missing? Antimicrobial Resistance and Infection Control 2(1): 17.

Pasricha J, Koessler T, Harbarth S, Schrenzel J, Camus V, Cohen G, Perrier A, Pittet D and Iten A. (2013b) Carriage of extended-spectrum beta-lactamase-producing enterobacteriacae among internal medicine patients in Switzerland. Antimicrobial Resistance and Infection Control 2(1): 20.

Piso RJ, Käch R, Pop R, Zillig D, Schibli U, Bassetti S, Meinel D and Egli A. (2017) A Cross-Sectional Study of Colonization Rates with Methicillin-Resistant Staphylococcus aureus (MRSA) and ExtendedSpectrum Beta-Lactamase (ESBL) and Carbapenemase-Producing Enterobacteriaceae in Four Swiss Refugee Centres. PLoS One 12(1): $\mathrm{e} 0170251$.

Vaud State Statistics. (2020) New asylum requests by nationality, Vaud, 1994 2019. Lausanne: Canton de Vaud. Available at: http://www.stat.vd.ch/ Default.aspx?DocID=7180\&DomId=1957 (accessed 26 April 2020). 